\title{
Dopant-Enhanced Vertical Alignment of Negative Liquid Crystals
}

\author{
Chien-Hui Wen \\ Benjamin Wu \\ Sebastian Gauza \\ Xiangyi Nie \\ Shin-Tson Wu
}

College of Optics and Photonics, University of Central Florida,

Orlando, Florida, USA

\begin{abstract}
A simple method for achieving high-contrast vertical alignment of high birefringence $(\Delta n \sim 0.28)$ negative dielectric anisotropic liquid crystal mixture using buffed polyimide substrates is demonstrated. By doping a positive, or non-polar dielectric anisotropic compound or mixture to the laterally difluorinated tolane liquid crystal host, an excellent vertical alignment was obtained. This alignment is stable throughout the entire nematic range. Its application for large screen liquid crystal televisions is considered.
\end{abstract}

Keywords: dopant effect; liquid crystal display; vertical alignment

\section{INTRODUCTION}

A vertical alignment (VA) cell $[1,2]$ has been used widely in liquid crystal display (LCD) devices because of its excellent contrast ratio. In the normal incidence, the contrast ratio is insensitive to the incident light wavelength, LC cell gap, and operating temperature [3]. For wide-view applications, e.g., LCD TVs and desktop monitors, multi-domain vertical alignment (MVA) [4], advanced super view [5], and patterned vertical alignment (PVA) [6] have been developed. To realize the useful electro-optic effects using longitudinal electric field, the VA cells require a negative dielectric anisotropic LC mixture, i.e., $\Delta \varepsilon=\varepsilon_{\|}-\varepsilon_{\perp}<0$.

The authors would like to thank Toppoly Optoelectronics (Taiwan) for the financial support.

Address correspondence to Shin-Tson Wu, College of Optics and Photonics, University of Central Florida, Orlando, Florida 32816-2700, USA. E-mail: swu@mail.ucf.edu 
For active matrix displays, high resistivity is another crucial requirement for obtaining high voltage holding ratio in order to avoid image flickering [7]. To achieve high resistivity, laterally fluorinated compounds are commonly employed [8].

To reduce image blurring for fast moving objects, fast response time is highly desirable for all LCD devices. A straightforward method for shortening response time is to reduce the LC cell gap while employing a higher birefringence $(\Delta \mathrm{n}>0.1)$ LC mixture in order to keep the necessary phase retardation. For infrared applications [9], the role of high birefringence and low viscosity becomes even more important because of the longer wavelength and reduced birefringence [10]. The recently developed one-drop-fill method allows a thinner cell gap $(\mathrm{d}<4 \mu \mathrm{m})$ to be fabricated while maintaining high manufacturing yield. Laterally $(2,3)$ difluorinated biphenyl, tolane [11], and terphenyl [12] LC materials are the natural choices to obtain high $\Delta \mathrm{n}$, large but negative $\Delta \varepsilon$, high resistivity, and good photo and thermal stabilities. However, these laterally difluoro compounds are rather difficult to align in a VA cell; a poor molecular alignment leads to a low contrast ratio and smeared threshold voltage.

In our experiments, we formulated a high birefringence negative $\Delta \varepsilon$ LC mixture, designated as UCF-A, using several laterally difluorinated tolane homologues [11]. As expected, UCF-A is difficult to align in a VA cell; its dark state has a severe light leakage between two crossed polarizers. We report a simple method for aligning the high $\Delta \mathrm{n}$ and negative $\Delta \varepsilon \mathrm{LC}$ mixtures. By doping a few percent of positive $\Delta \varepsilon$ [13] or non-polar compounds into the negative LC host, an excellent vertical alignment is obtained. The VA cell exhibits a superb contrast ratio, sharp threshold, and good thermal stability throughout its nematic range. We also study the possible mechanism by coating 5CB $(\Delta \varepsilon>0)$ onto the glass substrates before we fill the negative $\Delta \varepsilon$ LC mixture into the cell. This experiment supports our hypothesis that the positive or non-polar dopant serves as a monolayer for wetting the glass substrate and reducing the repulsive force between the polyimide (PI) alignment layer and the lateral difluoro LC compounds. In addition, this method is applicable to different PI materials no matter it is buffed or not.

\section{EXPERIMENT}

To prepare the VA cells, we spin-coated a commercial polyimide SE1211 (from Nissan Chemicals) on the ITO (Indium-tin-oxide)-glass substrates, baked the substrates at $80^{\circ} \mathrm{C}$ for 5 minutes and then $180^{\circ} \mathrm{C}$ for 1 hour. We then gently rubbed the ITO-glass with cloth in 
anti-parallel directions. The rubbing induced pretilt angle is about $87^{\circ}$. We also prepared some VA cells without rubbing because the PVA and MVA cells do not require rubbing.

The melting $\left(\mathrm{T}_{\mathrm{m}}\right)$ and clearing $\left(\mathrm{T}_{\mathrm{c}}\right)$ temperatures of UCF-A are $22.6^{\circ} \mathrm{C}$ and $133.6^{\circ} \mathrm{C}$, respectively. Although the $\mathrm{T}_{\mathrm{m}}$ of UCF-A is very close to the room temperature, the super cooling effect keeps the mixture in its nematic phase at room temperature for weeks. The dopant we selected for this study is a non-polar biphenyl-cyclohexane, abbreviated as $\mathrm{BCH}$. We prepared three mixtures, B, C, and D, with $10 \%, 20 \%$ and $30 \%$ of $\mathrm{BCH}$ in UCF-A, respectively.

To determine the alignment performance, we measured the voltage dependent transmittance (V-T) of all above mentioned mixtures. We filled a $5-\mu \mathrm{m}$ homeotropic cell with these mixtures, and the filled cell was placed between two crossed polarizers while the cell rubbing direction oriented at $45^{\circ}$ with respect to the linear polarizer. A He-Ne laser $(\lambda=633 \mathrm{~nm})$ was used as the light source. The light transmittance was measured by a photodiode detector (New Focus Model 2031) and recorded digitally by a data acquisition system (DAQ, PCI 6110) using LabVIEW. An ac voltage with $1 \mathrm{kHz}$ square waves was used to drive the LC cell. Without specification, all the measurements were processed at $\mathrm{T}=50^{\circ} \mathrm{C}$.

\section{RESULTS AND DISCUSSION}

To determine the alignment performance of the LC mixtures, we first measured the voltage dependent transmittance (V-T) curves of UCF-A at $\mathrm{T}=23^{\circ} \mathrm{C}$ and $50^{\circ} \mathrm{C}$. Results are shown in Figure 1 . At $\mathrm{T}=23^{\circ} \mathrm{C}$, UCF-A has a relatively severe light leakage in the voltage-off state, and its threshold behavior is smeared. When the temperature is increased to $50^{\circ} \mathrm{C}$, which is the typical operating temperature of a LCD projector, the light leakage is worsened. These results indicate UCF-A does not align well in the VA cell, and the pretilt angle has increased significantly as the temperature increases [14]. Poor alignment of UCF-A depolarizes the input linearly polarized light resulting in strong light leakage when it passes through the analyzer.

To enhance the alignment quality, a non-polar biphenyl-cyclohexane $(\mathrm{BCH})$ dopant was used. The dielectric anisotropy of $\mathrm{BCH}$ is $\sim 0$ because the terminal groups are all alkyls. We prepared three LC mixtures, designated as $\mathrm{A}, \mathrm{B}$, and $\mathrm{C}$, with 10,20 , and $30 \mathrm{wt} \%$ of $\mathrm{BCH}$ in UCF-A. Figure 2 plots the V-T curves of these three doped LC mixtures at $\mathrm{T}=50^{\circ} \mathrm{C}$. As the dopant concentration increases, the dark state is improved gradually. When we increased the $\mathrm{BCH}$ to $30 \mathrm{wt} \%$, the cell exhibits an excellent dark state and sharp threshold voltage. 


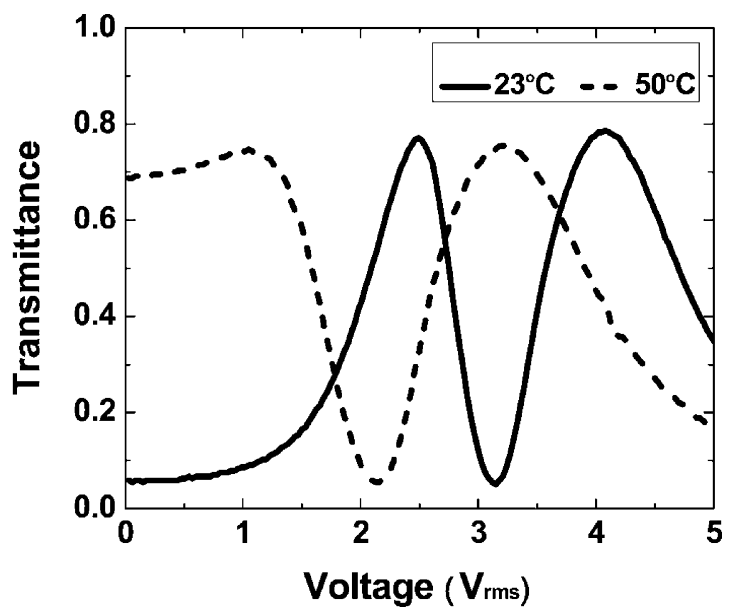

FIGURE 1 Voltage dependent transmittance curves of UCF-A in a VA cell at $\mathrm{T}=23^{\circ} \mathrm{C}$ (solid line) and $50^{\circ} \mathrm{C}$ (dashed line). $\lambda=633 \mathrm{~nm}$.

These results indicate the non-polar $\mathrm{BCH}$ compound helps to align the negative LC mixture in a VA cell. The required dopant percentage depends on the detailed molecular interactions between the alignment layer and the negative LC mixture. In this study, we find that $10 \%$ and $20 \%$ of $\mathrm{BCH}$ dopant is not enough to neutralize the strong

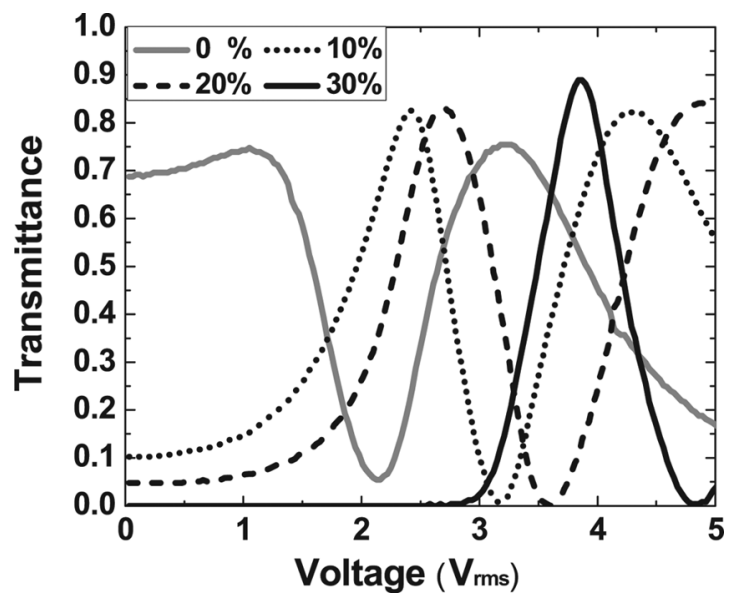

FIGURE 2 Voltage dependent transmittance curves of mixtures A (black dotted), B (black dashed) and C (black solid) in VA cells at T $=50^{\circ} \mathrm{C}$. V-T curve of UCF-A (gray line) is included as a reference. $\lambda=633 \mathrm{~nm}$. 
repulsive force between PI and UCF-A. However, at 30\%, excellent alignment is achieved. The melting point $\left(\mathrm{T}_{\mathrm{m}}\right)$ and clearing point $\left(\mathrm{T}_{\mathrm{c}}\right.$ ) of mixture $\mathrm{C}$ are $-16.3^{\circ} \mathrm{C}$ and $142.5^{\circ} \mathrm{C}$, respectively, and it presents nematice phase during our experiments. A drawback for doping too much non-polar compound is that its threshold voltage increases. Therefore, an optimal dopant concentration should be considered.

In addition to investigate the V-T curves, we also observed these rubbed VA cells under polarizing optical microscope. The rubbing direction of VA cell was oriented at $0^{\circ}$ with respect to the polarizer. Results are shown in Figure 3. Figure 3(a) represents the VA cell filled
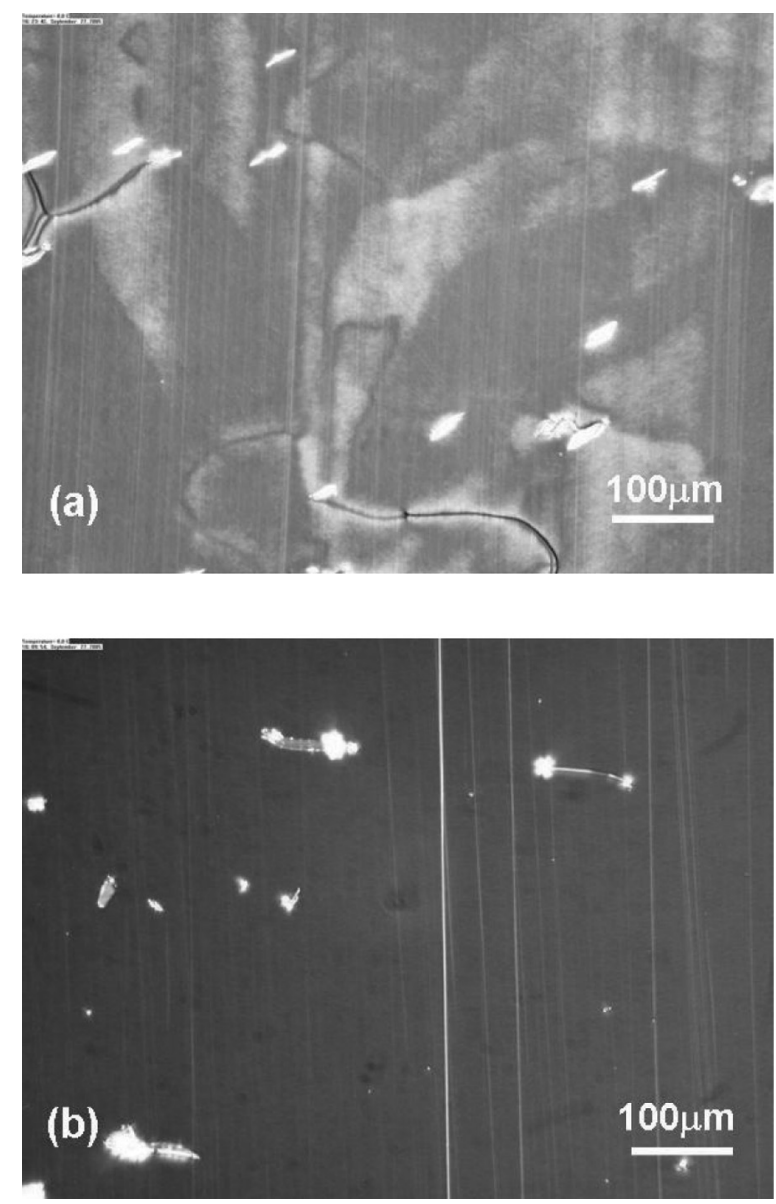

FIGURE 3 Microscope photos of a rubbed polyimide VA cell filled with (a) UCF-A and (b) mixture C under crossed polarizers. 
with UCF-A, while Figure 3(b) is for mixture C. The alignment of UCF-A is not uniform and the cell has a strong light leakage between two crossed polarizers. On the contrary, mixture C aligns uniformly and has a good dark state.

Besides the rubbed PI cell, we also tested our concepts using nonrubbed PI substrates and similar results were obtained. We prepared another two mixtures, mixture D and E, for this study. Mixture D is a binary eutectic mixture by mixing two lateral difluoro-terphenyl homologues. The terminal groups of these two homologues are $R_{1}=$ $\mathrm{C}_{2} \mathrm{H}_{5}, \mathrm{R}_{2}=\mathrm{C}_{4} \mathrm{H}_{9}$, and $\mathrm{R}_{1}=\mathrm{C}_{3} \mathrm{H}_{7}, \mathrm{R}_{2}=\mathrm{C}_{5} \mathrm{H}_{11}$. Mixture D consists of $35 \mathrm{wt} \%$ and $65 \mathrm{wt} \%$ of the -24 and -35 homologues, respectively. Mixture E contains $90 \%$ of mixture D and $10 \%$ of MLC-9200-000 (from Merck, $\Delta \varepsilon>0$ ). The nematic range of mixture $\mathrm{D}$ is from $23.6^{\circ} \mathrm{C}$ to $112.3^{\circ} \mathrm{C}$, but the supercooling effect extends the melting point down to $6.8^{\circ} \mathrm{C}$. Thus, the mixture stays liquid at room temperature. Figure 4(a) and (b) represent the microscope images (between crossed polarizers) of mixture $\mathrm{D}$ at $\mathrm{V}=0$ and $9 \mathrm{~V}_{\mathrm{rms}}$, respectively. Similarly,
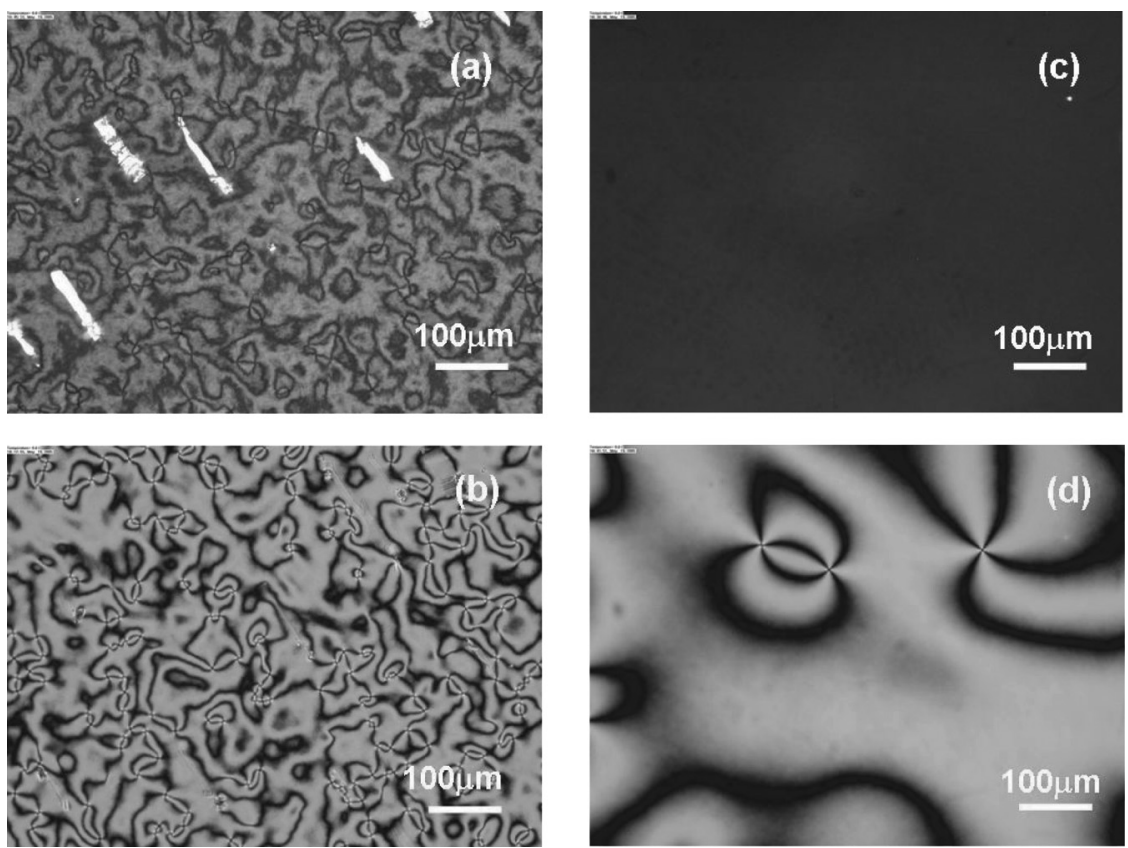

FIGURE 4 Microscope photos of non-rubbed polyimide VA cells filled with (a) mixture $\mathrm{D}, \mathrm{V}=0$, (b) mixture $\mathrm{D}, \mathrm{V}=9 \mathrm{~V}_{\text {rms }}$, (c) mixture $\mathrm{E}, \mathrm{V}=0$, and (d) mixture $\mathrm{E}, \mathrm{V}=9 \mathrm{~V}_{\mathrm{rms}}$ under crossed polarizers. 
Figure 4(c) and (d) represent the VA cell of mixture $\mathrm{E}$ at $\mathrm{V}=0$ and $9 \mathrm{~V}_{\text {rms }}$, respectively. In Figure $4(\mathrm{a})$ and (b), there are many small domains whose sizes are much smaller than those in Figure 4(d). These small patterns result in light leakage in the voltage-off state and low transmittance in a voltage-on state. On the contrary, Figure 4(c) shows an excellent dark state between two crossed polarizers. This indicates the doping method also improves the alignment of negative LC mixture in non-rubbed VA cell. The non-rubbed PI substrates have been used in multi-domain structures [15-16] for widening the viewing angle.

\section{POSSIBLE MECHANISM}

To study the possible mechanism of dopant effects on improving the alignment of negative $\Delta \varepsilon \mathrm{LC}$ mixture in a VA cell, we selected mixture $\mathrm{D}$ and $5 \mathrm{CB}$ for this study. We selected $5 \mathrm{CB}$ as the dopant because of its low clearing temperature $\left(35.3^{\circ} \mathrm{C}\right)$. We used two different methods to coat $5 \mathrm{CB}$ onto the glass substrate: 1 ) we vaporized $5 \mathrm{CB}$ onto the glass substrate and 2) we dissolved 5CB into a cyclohexane solution and then dripped this solution onto a glass substrate. In the first method, we filled a glass vial with $5 \mathrm{CB}$ and placed a glass substrate, which has the PI alignment layer, over the opening. We heated the $5 \mathrm{CB}$ to $\sim 120^{\circ} \mathrm{C}$ for 20 minutes, evaporating the $5 \mathrm{CB}$ onto the substrate, and then removed the glass substrate. We then repeated the process for another substrate and then assembled a cell using the two substrates coated with 5CB. The sealed cell was then injected with mixture D. We observed this LC cell under a polarizing optical microscope. Result is shown in Figure 5. Between crossed polarizers, the area coated with $5 \mathrm{CB}$ vapor appears darker than the adjacent uncoated areas, indicating that the coated 5CB indeed helps align mixture D.

In the second method, we prepared a $5 \mathrm{CB}$ solution in cyclohexane. The molar concentration of the $5 \mathrm{CB}$ solution was $5.3 \times 10^{-3} \mathrm{~mol} / \mathrm{L}$. We dripped the solution onto the glass substrate inside an oven $\left(\sim 90^{\circ} \mathrm{C}\right)$ in order to vaporize the cyclohexane, leaving just the $5 \mathrm{CB}$ on the substrate. We placed 10 drops, each time waiting for the cyclohexane to evaporate before placing another drop, in order to deposit enough 5CB molecules on the glass substrate. After being coated with the 5CB solution, the cell was sealed and injected with mixture D. Result is shown in Figure 6.

In Figure 6, the left upper corner is the area without 5CB, where the light leaks severely because of the poor LC alignment. The lower right corner is the area with the $5 \mathrm{CB}$ solution. The slightly diagonal lines are the rubbing direction. The 5CB solution, indeed, enhances the contrast ratio of a VA cell. 


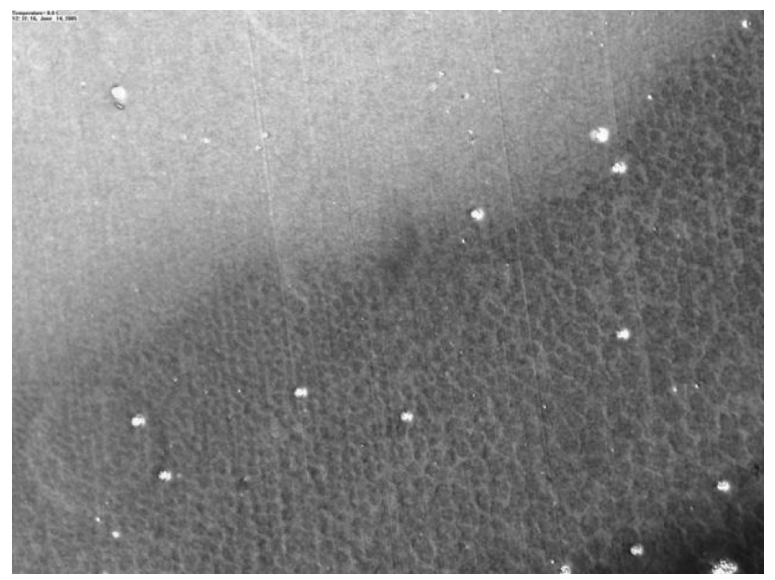

FIGURE 5 A microscope photo of a VA cell filled with mixture D under crossed polarizers. The PI alignment layer was over-coated with vaporized $5 \mathrm{CB}$ molecules before the cell was injected with mixture D. The dark area (bottom right corner) is the area coated with the $5 \mathrm{CB}$ vapor. A $20 \times$ magnification objective lens was used.

We found that, compared to Figure 5, the solvent method forms a larger and more uniform area. This is because the vaporized 5CB molecules could aggregate and cause non-uniformity, as shown in Figure 5.

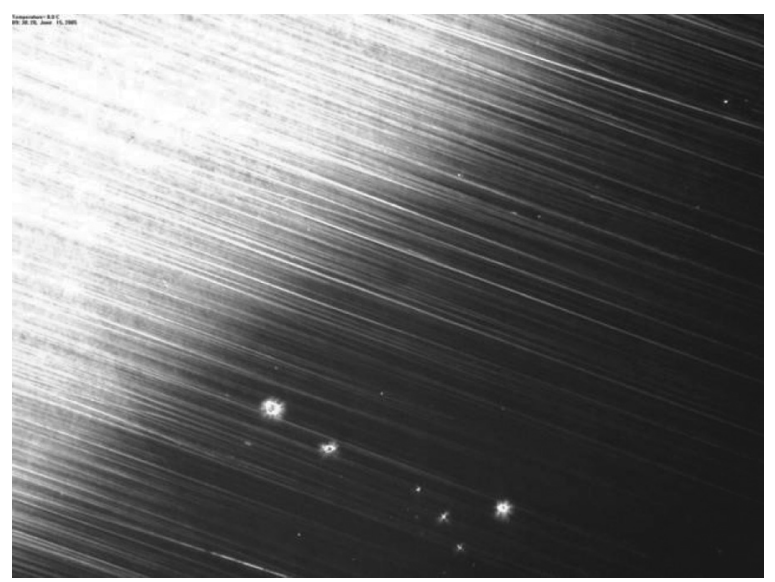

FIGURE 6 A microscope photo of VA cell under crossed polarizers. The surfaces of the VA cell were coated with 5CB dissolved in cyclohexane solution. The cell was then filled with mixture D. A $20 \times$ magnification objective lens was used. The bottom right corner is the area with $5 \mathrm{CB}$ solvent. 
From this surface treatment by coating $5 \mathrm{CB}$ on the glass substrate, we found the area with 5CB treatment has much better dark state than the others. Although we do not know the exact molecular structure of the PI we employed, we believe that the 5CB serves as a buffer layer for aligning the difluoro compounds and mixtures and it neutralizes the strong negative charge from the negative LC mixtures. The dopants reduce the repulsive force between polyimide and negative LC mixtures. This strong repulsive force results in a large pretilt angle (away from vertical direction), and causes light leakage in the dark state. This evidence was confirmed when we observed VA cell filled with UCF-A between two crossed polarizers under a light table. We saw a very colorful cell rather than one with a good dark state.

\section{CONCLUSION}

We have developed a new method for aligning the high birefringence, lateral difluorinated tolane mixture, UCF-A. By doping 10, 20, and $30 \mathrm{wt} \%$ of non-polar cyclohexane-biphenyl LC compound to the host negative LC mixtures, light leakage is suppressed gradually even at an elevated operating temperature. The required amount of dopant depends on the interaction between polyimide layer and LC mixture. We study the possible mechanism by coating $5 \mathrm{CB}(\Delta \varepsilon>0)$ on the glass substrate, and it supports our hypothesis. We also test this new method by using non-rubbed PI cells, and similar effects are observed. By carefully choosing the dopant, the mixture's figure-of-merit could be improved, in addition to a better alignment. A widespread application of this alignment method to different display devices is foreseeable.

\section{REFERENCES}

[1] Schiekel, M. F. \& Fahrenschon, K. (1971). Appl. Phys. Lett., 19, 391.

[2] Kahn, F. J. (1973). Appl. Phys. Lett., 22, 386.

[3] Wu, S. T. \& Yang, D. K. (2001). Reflective Liquid Crystal Displays, Wiley: New York.

[4] Takeda, A., Kataoka, S., Sasaki, T., Chida, H., Tsuda, H., Ohmuro, K., Sasabayashi, T., Koike, Y., \& Okamoto, K. (1998). Soc. Inform. Display Tech. Digest, 29, 1077.

[5] Ishii, Y., Mizushima, S., \& Hijikigawa, M. (2001). Soc. Inform. Display Tech. Digest, $32,1090$.

[6] Kim, S. (2004). Soc. Inform. Display Tech. Digest, 35, 760.

[7] Kirsch, P. \& Bremer, M. (2000). Angew. Chem. Int. Ed., 39, 4216.

[8] Reiffenrath, V., Krause, J., Plach, H. J., \& Weber, G. (1989). Liq. Cryst., 5, 159.

[9] Wu, S. T., Efron, U., \& Hess, L. D. (1984). Appl. Phys. Lett., 44, 1033. 
[10] Wu, S. T. (1986). Phys. Rev. A, 33, 1270.

[11] Wu, S. T., Hsu, C. S., \& Chen, J. M. (1997). Mol. Cryst. Liq. Cryst., 304, 441.

[12] Gray, G. W., Hird, M., \& Toyne, K. J. (1991). Mol. Cryst. Liq. Cryst., 204, 43.

[13] Wen, C. H., Gauza, S., Li, J., Wang, H., \& Wu, S. T. (2005). Liq. Cryst., 32, 643.

[14] Wen, C. H., Gauza, S., \& Wu, S. T. (2005). Appl. Phys. Lett., 87, 191909.

[15] Kim, K. \& Kim, S. (2003). Soc. Inf. Display Tech. Digest, 34, 1208.

[16] Okamoto, K. (2003). Proc. Int'l Display Manufacturing Conference, 143. 